\title{
Multipartite entanglement dynamics in a cavity
}

\author{
J G Amaro ${ }^{1}$, C Pineda ${ }^{2}$ \\ ${ }^{1}$ Facultad de Ciencias, Universidad Nacional Autónoma de México, México D.F. \\ 01000, México \\ ${ }^{2}$ Instituto de Física, Universidad Nacional Autónoma de México, México D.F. \\ 01000, México
}

\begin{abstract}
We study the dynamics of two kinds of entanglement, and there interplay. On one hand, the intrinsic entanglement within a central system composed by three two level atoms, and measured by multipartite concurrence; on the other, the entanglement between the central system and a cavity, acting as an environment, and measured with purity. Using dipole-dipole and Ising interactions between atoms we propose two Hamiltonians, a homogeneous and a quasi-homogeneous one. We find an upper bound for concurrence as a function of purity, associated to the evolution of the $W$ state. A lower bound is also observed for the homogeneous case. In both situations, we show the existence of critical values of the interaction, for which the dynamics of entanglement seem complex.

PACS numbers: 03.65.Yz,03.67.-a
\end{abstract}

\section{Introduction}

Entanglement was appreciated by Schrödinger not as "one but rather the characteristic trait of quantum mechanics" [1. Its study has opened its own field field [2, 3, that has remain active because the understanding of entanglement has proved valuable to comprehend the transition from the quantum to the classical world. Also, from a pragmatic point of view, to build ever more complex quantum technologies, one needs to tame its effects. Entanglement has been identified as a key resource in, say, quantum simulation and quantum computing [4.

Several theoretical developments have been devoted to understand the evolution of the entanglement of bipartite systems, both from theoretical, and an experimental point of view (see for example, [ $[, 6,7$, and many articles citing these ones). Nowadays, two party entanglement is routinely produced, controlled, studied and exploited in the laboratory. Moreover, it is well understood. Many aspects of multipartite entanglement, despite a huge effort, still remain as open questions (see for example 8] and [9]). In this paper we shall study the simplest case of multipartite entanglement, namely three qubits, and its interplay with bipartite entanglement, often associated with decoherence. For this, we use the simplest possible "reservoir" with an infinite spectrum: a harmonic oscillator.

Building upon [10] we generalize there model, to allow for more qubits, but retaining its simplicity. We consider three two-level atoms, coupled to each other via dipole and Ising interaction. Additionally, we consider a cavity which shall be regarded as an environment, to which the atoms are coupled to a single mode. Both the qubits and the cavity have its free dynamics. Except for very special cases, this 
model is not analytically solvable. However, number conservation will allow us to work on a finite Hilbert space, and perform numerics. We shall focus on the evolution of the entanglement withing the three qubits, and of those, with the cavity, as a measure of decoherence. More over, we shall also study the relation between these two very different quantities, via a generalized $C P$ (concurrence-purity) map [11, 6, 10,

Our paper is organized as follows. In section section 2 we introduce the model, and discuss some of its symmetries. We devote section section 3 to recall some aspects of entanglement that shall be discussed here. Special attention will be given to multipartite mixed entanglement, as quantified by the concurrence. In section 4 we present our findings, and we finish with some conclusion in section 5.

\section{The model}

We shall work in the Hilbert space associated to a harmonic oscillator, and three qubits. That is $\mathcal{H}=\mathcal{H}_{\mathrm{ho}} \otimes \mathcal{H}_{\mathrm{q}, 1} \otimes \mathcal{H}_{\mathrm{q}, 2} \otimes \mathcal{H}_{\mathrm{q}, 3}$ with $\mathcal{H}_{\mathrm{ho}}$ the Hilbert space of the oscillator, and the others, the Hilbert space of each of the three qubits $\left(\operatorname{dim} \mathcal{H}_{\mathrm{q}, \mathrm{i}}=2\right)$. The Hamiltonian that will determine the evolution of the system is

$$
\begin{aligned}
\hat{H}=\sum_{j=1}^{3} \frac{\Delta_{j}}{2} \hat{\sigma}_{z}^{(j)} & +\sum_{j=1}^{3} g_{j}\left(\hat{a} \hat{\sigma}_{+}^{(j)}+\text { h.c. }\right) \\
& +2 \sum_{j \neq k=1}^{3} \kappa_{j k}\left(\hat{\sigma}_{-}^{(j)} \hat{\sigma}_{+}^{(k)}+\text { h.c. }\right)+\sum_{j \neq k=1}^{3} J_{j k} \hat{\sigma}_{z}^{(j)} \hat{\sigma}_{z}^{(k)},
\end{aligned}
$$

in which we are using Pauli matrices $\hat{\sigma}_{x, y, z}^{(j)}$ acting in the $j$ th spin $1 / 2$ particle, the lowering and rising operators of an harmonic oscillator $\left(\hat{a}, \hat{a}^{\dagger}\right)$ and the rising and lowering operators of spin $1 / 2$ particle $j, \hat{\sigma}_{ \pm}^{(j)}=\hat{\sigma}_{x}^{(j)} \pm \mathrm{i} \hat{\sigma}_{y}^{(j)}$. We are also introducing several parameters, namely the energy splitting in each qubit $\left(\Delta_{j}\right)$, the intensity of their interaction with the harmonic oscillator $g_{j}$, the pairwise dipole-dipole interaction $\kappa_{j k}$ and $J_{j k}$ the Ising interaction. Intrinsic dynamics in the oscillator can be safely ignored using the appropriate interaction picture.

Note that despite the simplicity of the model, it will admit several very distinct dynamical configurations, ranging from: all qubits interacting with each other; a "line" configuration in which there is no interaction between qubits 1 and 3; a spectator configuration in which one qubit does not interact with the other qubits; and finally one in which all qubits are decoupled from each other. This can be controlled by setting to zero several of the coupling parameters $\kappa_{j k}$ and $J_{j k}$.

A very important feature, that allows us to treat the model, is that this Hamiltonian preserves the number of excitations, characterized by the operator

$$
\hat{N}=\frac{1}{2} \sum_{j=1}^{3} \hat{\sigma}_{z}^{(j)}+\hat{a}^{\dagger} \hat{a}+\frac{3}{2} \mathbb{1} .
$$

One can thus write the Hamiltonian in block-diagonal form in a suitable basis. We 
take this basis to be

$$
\begin{aligned}
\left|\phi_{0}^{(n)}\right\rangle & =|n\rangle|000\rangle, & & \left|\phi_{4}^{(n)}\right\rangle=|n-2\rangle|110\rangle, \\
\left|\phi_{1}^{(n)}\right\rangle & =|n-1\rangle|001\rangle, & & \left|\phi_{5}^{(n)}\right\rangle=|n-2\rangle|101\rangle, \\
\left|\phi_{2}^{(n)}\right\rangle & =|n-1\rangle|010\rangle, & \left|\phi_{6}^{(n)}\right\rangle & =|n-2\rangle|011\rangle, \\
\left|\phi_{3}^{(n)}\right\rangle & =|n-1\rangle|100\rangle, & \left|\phi_{7}^{(n)}\right\rangle & =|n-3\rangle|111\rangle,
\end{aligned}
$$

so that $\hat{N}\left|\phi_{i}^{(n)}\right\rangle=n\left|\phi_{i}^{(n)}\right\rangle$. We are using the convention in which $|1\rangle$ means an excitation and $|0\rangle$ means no excitation, so $\sigma_{+}|0\rangle=|1\rangle$. Note that, when using matrix representation, even though their is a one to one correspondence with the state of the qubits, there is additional information regarding the state of the harmonic oscillator, via de superindex $(n)$. The order chosen for the basis will allow us to write the partial trace in a particularly nice fashion. Given the matrix representations of an arbitrary mixed state $\rho$ acting in the subspace of $n$ excitations, we can write

$$
\operatorname{tr}_{\mathrm{osc}} \rho=\left(\begin{array}{cccccccc}
\rho_{00} & 0 & 0 & 0 & 0 & 0 & 0 & 0 \\
0 & \rho_{11} & \rho_{12} & \rho_{13} & 0 & 0 & 0 & 0 \\
0 & \rho_{21} & \rho_{22} & \rho_{23} & 0 & 0 & 0 & 0 \\
0 & \rho_{31} & \rho_{32} & \rho_{33} & 0 & 0 & 0 & 0 \\
0 & 0 & 0 & 0 & \rho_{44} & \rho_{45} & \rho_{46} & 0 \\
0 & 0 & 0 & 0 & \rho_{54} & \rho_{55} & \rho_{56} & 0 \\
0 & 0 & 0 & 0 & \rho_{64} & \rho_{65} & \rho_{66} & 0 \\
0 & 0 & 0 & 0 & 0 & 0 & 0 & \rho_{77}
\end{array}\right) .
$$

A generic block of $n \geq 3$ excitations given by

$$
H^{(n)}=\left(\begin{array}{cc}
H_{A A}^{(n)} & H_{A B}^{(n)} \\
\left(H_{A B}^{(n)}\right)^{\dagger} & H_{B B}^{(n)}
\end{array}\right)-\sum_{j \neq k=1}^{3} J_{j k} \mathbb{1}
$$

with the first block being

$$
H_{A A}=\left(\begin{array}{cccc}
2 J & g_{1}^{+} & g_{2}^{+} & g_{3}^{+} \\
g_{1}^{+} & \delta_{1}^{+} & \kappa_{12} & \kappa_{13} \\
g_{2}^{+} & \kappa_{12} & \delta_{2}^{+} & \kappa_{23} \\
g_{3}^{+} & \kappa_{13} & \kappa_{23} & \delta_{3}^{+}
\end{array}\right)-\sum_{j=1}^{3} \Delta_{j} \mathbb{1},
$$

if $g_{i}^{ \pm}=g_{i} \sqrt{n \pm 1}, \delta_{i}^{ \pm}=2 J_{\neq i} \pm 2 \Delta_{i}+$, and $\mathrm{T}_{\Delta, J}=\sum_{i} J_{\neq i}-\Delta_{i}$. Note that we use the subindex $\neq i$ to refer to a pair of subindices, that are different from $i$ and from each other. The off-diagonal matrix is

$$
H_{A B}=\left(\begin{array}{cccc}
0 & 0 & 0 & 0 \\
0 & g_{3}^{0} & g_{2}^{0} & 0 \\
g_{3}^{0} & 0 & g_{1}^{0} & 0 \\
g_{2}^{0} & g_{1}^{0} & 0 & 0
\end{array}\right)
$$

with $g_{i}^{0}=g_{i} \sqrt{n}$. Finally, the second diagonal block is given by

$$
H_{B B}=\left(\begin{array}{cccc}
\delta_{3}^{-} & \kappa_{12} & \kappa_{13} & g_{1}^{-} \\
\kappa_{12} & \delta_{2}^{-} & \kappa_{23} & g_{2}^{-} \\
\kappa_{13} & \kappa_{23} & \delta_{1}^{-} & g_{3}^{-} \\
g_{1}^{-} & g_{2}^{-} & g_{3}^{-} & 2 J
\end{array}\right)+\sum_{j=1}^{3} \Delta_{j} \mathbb{1}
$$


For smaller values of $n$, the matrix will be the first block of 1,4 , and 7 states corresponding to (5), for $n=0,1,2$ respectively.

This model has a big number of parameters. A simplification, would be to consider a homogeneous situation, that is, all $\kappa_{i j}=\kappa, J_{i j}=J, g_{i}=g$ and $\Delta_{i}=\Delta$. Moreover, we shall set the detuning to be $\Delta=0$ and the coupling to the oscillator to $g=1$ in our numerical calculations, so that we have the homogeneous Hamiltonian

$$
\hat{H}_{\kappa, J}^{\mathrm{h}}=\sum_{j=1}^{3}\left(\hat{a} \hat{\sigma}_{+}^{(j)}+\text { h.c. }\right)+2 \kappa \sum_{j \neq k=1}^{3}\left(\hat{\sigma}_{-}^{(j)} \hat{\sigma}_{+}^{(k)}+\text { h.c. }\right)+J \sum_{j \neq k=1}^{3} \hat{\sigma}_{z}^{(j)} \hat{\sigma}_{z}^{(k)} \text {. }
$$

In order to appreciate the effect of homogeneity in the Hamiltonian, we also consider a slightly inhomogeneous case: we change one dipole-dipole interaction, to obtain the quasi-homogeneous Hamiltonian

$$
\hat{H}_{\kappa, J}^{\mathrm{qh}}=\hat{H}_{\kappa, J}^{\mathrm{h}}+\kappa\left(\hat{\sigma}_{-}^{(1)} \hat{\sigma}_{+}^{(2)}+\text { h.c. }\right) \text {. }
$$

Several other simple ways of introducing the inhomogeneity are also available, however, this one displays very clearly the effects we want to underline in this work.

Note that one further consideration, in the case of the homogeneous case, could be done: the Hamiltonian is invariant under rotations. In this case, the rotation operator $\hat{R}$ that acts on the computational basis as $\hat{R}|n\rangle\left|i_{1} i_{2} i_{3}\right\rangle=|n\rangle\left|i_{3} i_{1} i_{2}\right\rangle, i_{j}= \pm$, has three eigenvalues, namely $\exp (2 \pi \mathrm{i} j / 3)$, with $j=0,1,2$. Let us define the vectors

$$
\begin{aligned}
& \left|\varphi_{0}^{(n, k)}\right\rangle=\alpha^{k}\left|\phi_{1}^{(n)}\right\rangle+\alpha^{2 k}\left|\phi_{2}^{(n)}\right\rangle+\alpha^{2 k}\left|\phi_{3}^{(n)}\right\rangle, \\
& \left|\varphi_{1}^{(n, k)}\right\rangle=\alpha^{k}\left|\phi_{4}^{(n)}\right\rangle+\alpha^{2 k}\left|\phi_{5}^{(n)}\right\rangle+\alpha^{2 k}\left|\phi_{6}^{(n)}\right\rangle,
\end{aligned}
$$

$(k=0,1,2)$ with $\alpha=\exp (2 \pi \mathrm{i} / 3)$ and the additional $\left|\varphi_{2}^{(n, 0)}\right\rangle=\left|\phi_{0}^{(n)}\right\rangle$ and $\left|\varphi_{3}^{(n, 0)}\right\rangle=$ $\left|\phi_{7}^{(n)}\right\rangle$. These are eigenvectors of $\hat{R}$ with eigenvalues $\alpha^{k}$, that is, $\hat{R}\left|\varphi_{l}^{(n, k)}\right\rangle=\alpha^{k}\left|\varphi_{l}^{(n, k)}\right\rangle$. This lead to a splitting of the subspace in spaces of dimension 4, 2 and 2 . Very lengthy expressions however, make it very difficult to extract the general behaviour, and one would be forced to fall back to numerics. On the other hand, if one wishes to restrict to the symmetric subspace, the machinery of the Dicke states could be used, where some analytical results, regarding entanglement, are available [12, 13].

\section{Multipartite entanglement}

The notion of entanglement is defined using separability. Separable pure states $|\psi\rangle$ are those for which, in a multipartite Hilbert space $\mathcal{H}=\otimes_{i} \mathcal{H}_{i}$, can be written as a tensor product. That is, $|\psi\rangle \in \mathcal{H}$ is separable if $|\psi\rangle=\otimes_{i}\left|\psi_{i}\right\rangle$, with $\left|\psi_{i}\right\rangle \in \mathcal{H}_{i}$. Entangled states are those with are not a mixture of pure separable states. The problem of determining if a mixed state is or not entangle is difficult.

However, for the special case of pure bipartite states, all the information regarding entanglement is encoded in its Schmidt coefficients. One can choose any convex function of the Schmidt coefficients, being the von Neumann entropy and purity the most common choices. We shall use purity, defined for mixed states as

$$
P(\rho)=\operatorname{tr} \rho^{2}
$$


due to its algebraic simplicity. Purity can be regarded as an entanglement measure, provided that the state over which purity is calculate is given by

$$
\rho=\operatorname{tr}_{\mathrm{env}}|\psi\rangle\langle\psi| .
$$

If no a priori information is given about $\rho$, purity is simply a measure of mixedness. The value of purity ranges between $1 / N \leq P \leq 1$, where $N$ is the dimension of the Hilbert space in which $\rho$ acts, the minimum value corresponding to the maximally mixed state, and the maximum to a pure one.

Characterizing multipartite entanglement on the other hand proves more challenging, as even a unique maximally entangled state does not exist for more than two parties [14. Most measures provide either well founded physical grounds, or numerically simple recipes. A convenient compromise is given by the multipartite concurrence. This measure, a generalization of the two party concurrence [15, is inspired in the symmetry properties of pure states. That is, in expected values of projections over antisymmetric subspaces. The detailed construction is out of reach within this presentation, but it reduces to the very simple form [16] for pure states:

$$
C(|\psi\rangle)=\frac{1}{2^{N / 2-1}} \sqrt{\left(2^{N}-2\right)-\sum_{i} \operatorname{tr} \rho_{i}^{2}}
$$

where the index $i$ runs over all proper subsets of particles, except for the empty set. For example, in the three particle case, we must consider all tree partitions in which the particles can be divided, that is particle $\mathrm{A}$ against $\mathrm{BC}$; $\mathrm{B}$ with $\mathrm{AC}$; and $\mathrm{C}$ with AB. Thus, $C(|\psi\rangle)^{2} \propto\left\langle 1-P\left(\rho_{i}\right)\right\rangle$ where here, the average $\langle\cdot\rangle$ is taken again over all non-trivial subsets particles, thus relating the measure with the entanglement over all possible partitions.

This measure, as presented above, will not suit our purposes, as it is defined only for pure states. We shall use the convex roof construction, in which the measure is averaged over a particular realization of our mixed state. Say, our state $\rho=\sum_{i} p_{i}\left|\psi_{i}\right\rangle\left\langle\psi_{i}\right|$, with normalized $\left|\psi_{i}\right\rangle$ and positive $p_{i}$ for all $i$. Then, we associate with this particular realization of $\rho$ the measure $\sum_{i} p_{i} C\left(\left|\psi_{i}\right\rangle\right)$. The measure is obtained finding the realization that minimizes such expression. This would mean, if one thinks about entanglement as a resource, the cheapest way of realizing such ensemble. Summarizing, we define

$$
C(\rho)=\inf _{p_{i},\left|\psi_{i}\right\rangle} \sum_{i} p_{i} C\left(\left|\psi_{i}\right\rangle\right),
$$

with $\rho=\sum_{i} p_{i}\left|\psi_{i}\right\rangle\left\langle\psi_{i}\right|, p_{i}>0$, and $\left\langle\psi_{i} \mid \psi_{i}\right\rangle=1$. Even though this is a very meaningful definition, the process of exact evaluation is normally difficult, as the landscape can be very complex. Upper bounds can be easily obtained using gradient methods to numerically optimize (16), and also simple, but useful lower algebraic bounds are available. In particular, we shall use the method of quasi-pure approximation [17. It is derived approximating a multi-matrix representation of a mixed state with a single matrix that captures the first few order terms in expressions involving the Schmidt coefficients of $\rho$. We underline that this is not only an approximation, but also a lower bound, reasonably tight even for some states close to the maximally mixed state [16, 18. To obtain this bound, no optimization procedure is involved, and only the diagonalization of a matrix of the same size as $\rho$ is needed. A detailed description requires some technicalities, that we do not wish to introduce. Instead, we refer the 

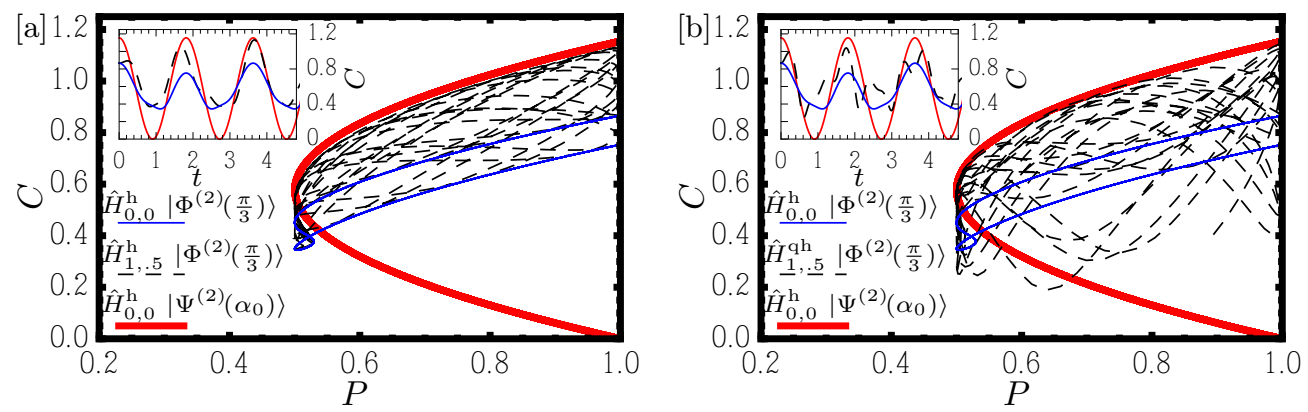

Figure 1. Parametric plot of concurrence and purity, for initial states of the form (17) and 18, with Hamiltonians 9 and 10 and an initial number of excitations of $n=1$. The red curve corresponds to three non-interacting atoms $(\kappa=J=0)$, initially in a $\mathrm{W}$ state. The black dashed curve shows the behaviour of three interacting atoms, with $\kappa=1$ and $J=0.5$, initially in a pure but not maximally entangled state $(\alpha=\pi / 3)$. The homogeneous 9 and quasihomogeneous 10 configurations are displayed in [a], and in [b] respectively. The blue curve shows the evolution of the decoupled system, with the same initial state as in the black dashed curve. All curves were parametrized by time up to $t=20$. The insets in displays the corresponding evolution of concurrence as function of time.

reader to 16. Some properties of the concurrence should be highlighted: it is invariant under unitary local operations, it vanishes only for completely separable states, and has the nice scaling property that $C(|\psi\rangle\langle\psi|\otimes| \phi\rangle\langle\phi|)=C(|\phi\rangle\langle\phi|)$ if $|\psi\rangle$ belongs to the Hilbert space of just one of the particles. Finally, the bound coincides with the concurrence for pure states.

The quasi-pure approximation suits very well our needs, where repeated evaluation of such a quantity is required, for many parameters and times. Moreover as we shall explore qualitative properties, so the small errors inherent in this approximation can be ignored.

\section{Results}

In this section we calculate the entanglement and purity as a function of time, and how they depend on each other. We consider two families of initial product states with the condition that any member of the family must belong to the eigenspace characterized by a fixed eigenvalue of the operator $\hat{N}$.

While the first family corresponds to the normalized product state with $n-1$ photons in the cavity and the superposition of the states $|001\rangle$ and $|010\rangle$,

$$
\left|\Phi^{(n)}(\alpha)\right\rangle=|n-1\rangle \otimes(\sin \alpha|001\rangle+\cos \alpha|010\rangle) .
$$

This states have no genuine tripartite entanglement, as one of the parties has a uncorrelated state, and the others share bipartite entanglement parametrized by $\alpha$. Its concurrence is $C\left(\left|\Phi^{(n)}(\alpha)\right\rangle\right)=\sin (2 \alpha)$. The second family corresponds to the superposition of the states $|001\rangle,|010\rangle$ and $|100\rangle$,

$$
\left|\Psi^{(n)}(\alpha)\right\rangle=|n-1\rangle \otimes\left(\frac{\sin \alpha}{\sqrt{2}}|001\rangle+\cos \alpha|010\rangle+\frac{\sin \alpha}{\sqrt{2}}|100\rangle\right) .
$$



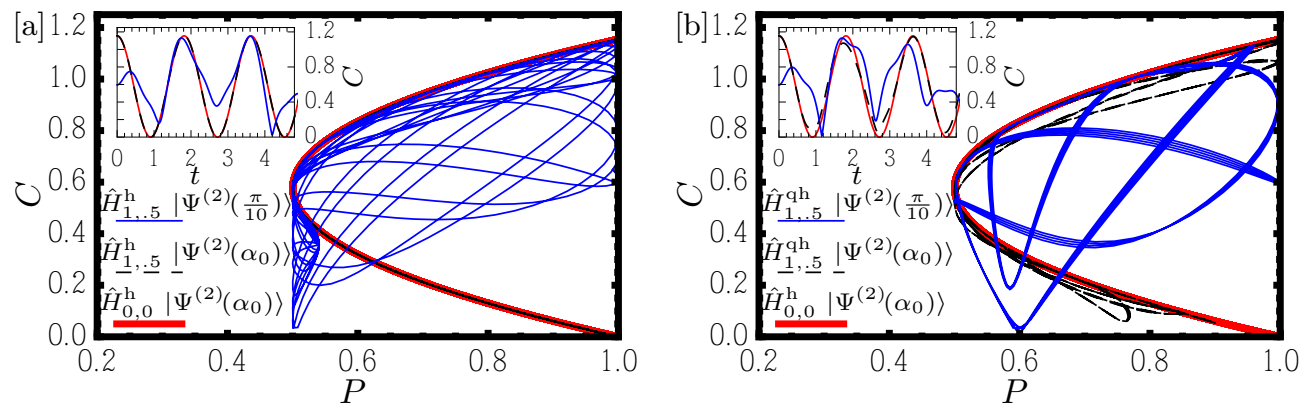

Figure 2. Same conventions as in figure 1. except that the initial states for the interacting case are given by 18 . The parameters are identical to the aforementioned figure.

This states do have genuine tripartite entanglement, as can be seen by setting $\alpha=\alpha_{0}=\arctan \sqrt{2}$ for which we retrieve the $|W\rangle$ state. Moreover, its concurrence is

$$
C\left(\left|\Psi^{(n)}(\alpha)\right\rangle\right)=\frac{\sin \alpha}{\sqrt{2}} \sqrt{5+3 \cos (2 \alpha)},
$$

with a maximum given by $C\left(\left|\Psi^{(n)}\left(\alpha_{0}\right)\right\rangle\right)=2 / \sqrt{3} \approx 1.15$. However, in both cases we can range from a totally separable state for $\alpha=0$ to a maximally entangled state for some critical $\alpha$. These families of states also have the important characteristic that will remain pure when the oscillator is traced out. The system evolves with the unitary evolution generated by Hamiltonian (1), and the state of the three qubits is given at time $t$ by

$$
\rho(t)=\operatorname{tr}_{\mathrm{ho}} \mathrm{e}^{-\mathrm{i} t \hat{H}}|\psi(0)\rangle\langle\psi(0)| \mathrm{e}^{\mathrm{i} t \hat{H}},
$$

where $|\psi(0)\rangle$ is the initial state of the system, which will be taken as either $\left|\Phi^{(n)}(\alpha)\right\rangle$ or $\left|\Psi^{(n)}(\alpha)\right\rangle$. One can then study the evolution of concurrence and purity, by studying $C(t)=C(\rho(t))$ and $P(t)=P(\rho(t))$ respectively. Moreover, we shall study an homogeneous case, in which all $J_{i j}=J$ and all $\kappa_{i j}=\kappa$.

In figures 1 and 2 we present concurrence against purity with time as a parameter, that is, the so called $C P$ plane. We studied in these figures the sector $n=1$, but the conclusions here drawn can be extended to higher excitation numbers, unless we explicitly say otherwise. We also show in the inset the evolution of concurrence. We fix the Hamiltonians to the homogeneous and quasi-homogeneous with parameters $J=0.5$ and $\kappa=1$. That is, using the notation of $(9)$ and $(10)$, we use $H_{1,0.5}^{\mathrm{h}}$ and $H_{1,0.5}^{\mathrm{qh}}$. In all cases an important benchmark shall be considered, namely a maximally mixed state $\left|\Psi^{(n)}\left(\alpha_{0}\right)\right\rangle \propto|001\rangle+|010\rangle+|100\rangle$, evolved with an interaction free model, that is, $H_{0,0}^{\mathrm{h}}$. This is plotted as a thick red curve.

In figure 1 we study initially non-maximally entangled states, both with and without interaction. In both figures 1, one can note that the red curve serves as an upper bound for the evolution of the states without maximal initial entanglement, similar to the two atom case [10]. Remarkably, for three atoms, the dynamics of the non-interacting case is a lower bound in the homogeneous case [a] (as in the two qubit case), whereas that is no longer the case when the system is not homogeneous [b]. We underline here that in all cases studied (and not shown), namely other parameters, and excitation numbers, these observations hold. The behaviour of concurrence and 

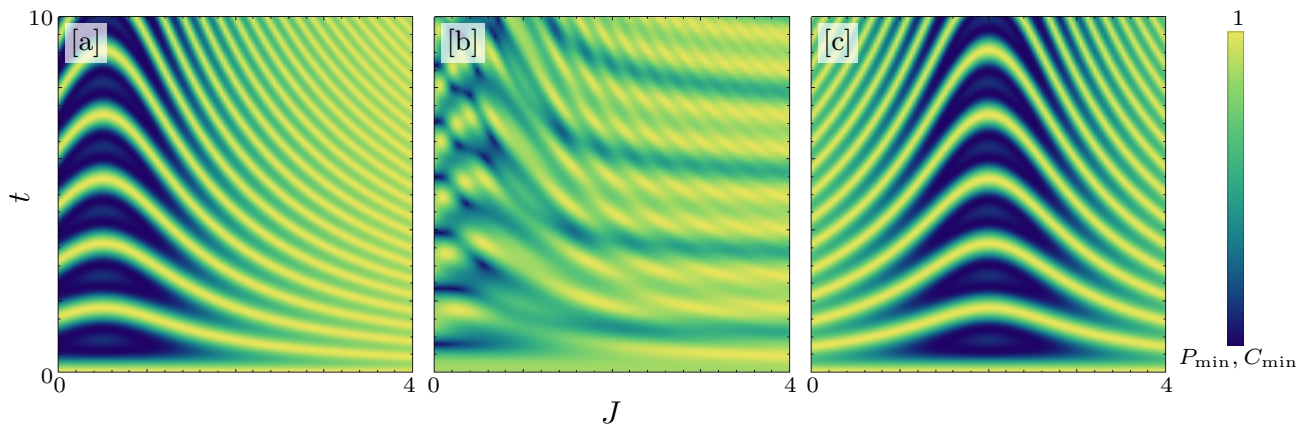

Figure 3. In these set of figures, we study the behaviour of purity and concurrence (as colour density, in arbitrary units) with the Ising interaction (in the $x$ axis) and time (in the $y$ axis) for the initial state $\left|\Phi^{(2)}(\pi / 4)\right\rangle$ and the Hamiltonian $\hat{H}_{\kappa, J}^{\mathrm{h}}$. In [a] we show purity, with $\kappa=1$, and observe no complex behaviour. Concurrence, plotted in [b], mimics this behaviour of purity, with added complexity. A larger dipole-dipole coupling $(\kappa=4)$, shown in [c], displaces the bump observed in [a], to larger values of $J$.

purity with time (displayed in the inset) will be discussed later, when we have a global picture, with respect to the parameters and time. However, it can be noted that the non homogeneous case display a richer behaviour.

In figure 2 we study the effect of interactions on both maximally entangled initial states $\left|\Psi^{(n)}\left(\alpha_{0}\right)\right\rangle$, and partially entangled states $\left|\Psi^{(n)}(\pi / 10)\right\rangle$. In this case, the behaviour of states which have initially maximum entanglement, with already large interaction, closely resemble the case with no interaction. In particular, for the homogeneous Hamiltonian the two curves coincide, while for the quasi-pure Hamiltonian the non interacting case acts as an imperfect, but very good guide. This is not the case if we start with a state with smaller entanglement. The red curve acts only as an upper bound for the interacting case. For larger values of the Ising interaction the dynamics display an oscillatory behaviour with almost the same periodicity. These observations are robust with respect to varying the systems and the total number of excitations.

In order to give a general view of the dynamics of purity and concurrence we proposed a density diagram display in figures 3 and 4 . There, concurrence and purity are colour coded for several values of the Ising interaction, for a given time span, under the influence of the homogeneous Hamiltonian. The initial state chosen was $\left|\Psi^{(2)}(\alpha=\pi / 4)\right\rangle$.

In figure 3 we compare the dynamics of purity (figures $3[\mathrm{a}, \mathrm{c}]$ ) and concurrence (figure $3 \mathrm{~b}$ b]). Indeed, concurrence mimics the behaviour of purity, with some additional nodes, caused possibly by the internal dynamics of the three qubits.

Purity displays a regular behaviour, with some some oscillations that seem to be independent of $J$ for large or small values of $J$. However, a critical area (around $J=0.5$ for $\kappa=1$, figure $3[\mathrm{a}]$ ) is clearly present. Varying the dipoledipole coupling shifts this critical value of $J$ to the right (figure $3[\mathrm{~b}]$ ). For higher excitation numbers, this region shows higher complexity, whereas the regular areas remain largely unchanged (see figure 4. As concurrence mimics the behaviour of purity, this observations are also valid for the internal entanglement. A remarkable fact is also seen: the existence of critical points seems to agree for all cases, for the 


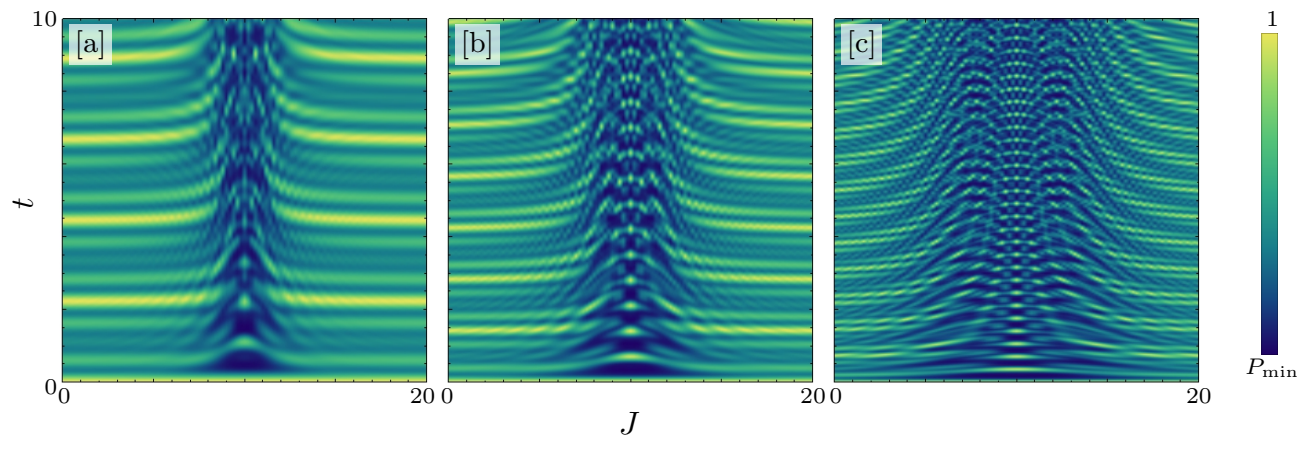

Figure 4. In these set of figures, we study the behaviour of purity with the Ising interaction (in the $x$ axis) and time (in the $y$ axis), for a varying number of total excitations $\left|\Phi^{(2,5,20)}(\pi / 4)\right\rangle$ in [a], [b] and [c] respectively, evolved with $\hat{H}_{20, J}^{\mathrm{h}}$. The region with complex behaviour increases with the number of excitations.

density diagrams (figures 3 and 4 .

\section{Conclusions and outlook}

In the present work we studied the entanglement dynamics of three interacting two level atoms inside a cavity with dipole-dipole and Ising interactions. Entanglement within the atoms is measured by concurrence, and of the atoms with the cavity, is measured by purity. Despite measuring totally different properties of the system, concurrence and purity are quite related. In particular in a concurrence-purity plane for given state, concurrence is bounded by above, by the curve described by a non interactive system, and initially in a $\mathrm{W}$ state. We believe that this upper bound is closely relate with monogamy of entanglement where entanglement cannot be freely shared among multiple parties [19]. Recall that our system is a four-partite state, namely three qubits and a bath in which entanglement must be considered, in this setting, not as a tripartite problem. At this respect, our analysis contributes to the existence of a hierarchy of strong monogamy (SM) inequalities (as propose by Regula et al. 20 ), or alternatively viewing multipartite entanglement from the point of view of frustration 21. In the homogeneous case, a lower bound, drawn by the evolution of the same initial state, but with a non-interacting Hamiltonian is also apparent. The inhomogeneous case, however, has not a simple lower bound. In fact, for this lower bound, calculations could be pursued using the Dicke States and the symmetric subspace, where the block-diagonal Hamiltonian and the basis, resembles the two qubit case. In the present work, this bound is very sensitive to the presence of a small perturbation, so this suggests a strong connection with the symmetric properties of the Dicke basis. A deeper study of these bound might prove useful in the context of mutipartite entanglement. Finally, we presented a global view for the dynamics of the concurrence and purity as function of time and Ising interaction, and show that there is a translation of the intervals where the dynamics exhibits complexity, when the dipole-dipole interaction is increasing. 


\section{Acknowledgments}

Support by the projects CONACyT 153190 and UNAM-PAPIIT IA101713 and IN111015 is acknowledged. Numerics were done building upon programs developed by Florian Minter and André Carvalho, and stimulating discussions with Thomas Seligman and Mauricio Torres are acknowledged.

\section{References}

[1] E. Schrödinger and M. Born. Discussion of Probability Relations between Separated Systems. Mathematical Proceedings of the Cambridge Philosophical Society, 31(04):555, October 1935.

[2] R. Horodecki, P. Horodecki, M. Horodecki, and K. Horodecki. Quantum entanglement. Rev. Mod. Phys., 81:865-942, Jun 2009.

[3] F. De Martini and F. Sciarrino. Colloquium. Rev. Mod. Phys., 84:1765-1789, Dec 2012.

[4] K. Modi, A. Brodutch, H. Cable, T. Paterek, and V. Vedral. The classical-quantum boundary for correlations: Discord and related measures. Rev. Mod. Phys., 84:1655-1707, Nov 2012.

[5] T. Konrad, F. de Melo, M. Tiersch, C. Kasztelan, A. Aragao, and A. Buchleitner. Evolution equation for quantum entanglement. Nat. Phys., 4(4):99-102, February 2008.

[6] C. Pineda and T. H. Seligman. Evolution of pairwise entanglement in a coupled $n$-body system. Phys. Rev. A, 73:012305, Jan 2006.

[7] J.-S. Xu, C.-F. Li, C.-J. Zhang, X.-Y. Xu, Y.-S. Zhang, and G.-C. Guo. Experimental investigation of the non-markovian dynamics of classical and quantum correlations. Phys. Rev. A, 82:042328, Oct 2010.

[8] L. Clarisse. The distillability problem revisited. Quantum Information and Computation, 6:539$560,2006$.

[9] N. Gisin. Bell inequalities: Many questions, a few answers. In Quantum Reality, Relativistic Causality, and Closing the Epistemic Circle, volume 73 of The Western Ontario Series in Philosophy of Science, pages 125-138. Springer Netherlands, 2009.

[10] J. M. Torres, E. Sadurní, and T. H. Seligman. Two interacting atoms in a cavity: exact solutions, entanglement and decoherence. J. Phys. A, 43(19):192002, 2010.

[11] Ziman. M. and V. Buzek. Concurrence versus purity: Influence of local channels on bell states of two qubits. Phys. Rev. A, 72(5):052325, 2005.

[12] Tsubasa I., Toshihiko S., Izumi T., and Nobuhiro Y. Exchange symmetry and multipartite entanglement. Phys. Rev. A, 78:052105, Nov 2008.

[13] S Campbell, M S Tame, and M Paternostro. Characterizing multipartite symmetric dicke states under the effects of noise. New J. Phys., 11(7):073039, 2009.

[14] W. Dür, G. Vidal, and J. I. Cirac. Three qubits can be entangled in two inequivalent ways. Phys. Rev. A, 62:062314, Nov 2000.

[15] W. K. Wootters. Entanglement of formation of an arbitrary state of two qubits. Phys. Rev. Lett., 80(10):2245, 1998

[16] F. Mintert, A. R. R. Carvalho, K. Marek, and A. Buchleitner. Measures and dynamics of entangled states. Phys. Rep., 415(4):207 - 259, 2005.

[17] F. Mintert and A. Buchleitner. Concurrence of quasipure quantum states. Phys. Rev. A, 72:012336, Jul 2005.

[18] F. Mintert and A. Buchleitner. Observable entanglement measure for mixed quantum states. Phys. Rev. Lett., 98:140505, Apr 2007.

[19] V. Coffman, J. Kundu, and W. Wootters. Distributed entanglement. Phys. Rev. A, 61:052306, Apr 2000.

[20] B. Regula, S. Di Martino, S. Lee, and G. Adesso. Strong monogamy conjecture for multiqubit entanglement: The four-qubit case. Phys. Rev. Lett., 113:110501, Sep 2014.

[21] P Facchi, G Florio, U Marzolino, G Parisi, and S Pascazio. Multipartite entanglement and frustration. New Journal of Physics, 12(2):025015, 2010. 